\title{
Pré- et probiotiques
}

Qui eut parié il y a dix ans sur l'avenir de la flore intestinale. Et pourtant, elle est montée en grade dans l'estime qu'en ont les physiologistes, les nutritionnistes, les chercheurs et les médecins de tous horizons : gastroentérologues, allergologues, immunologiste et "obésologues" ! Il n'y a qu’à voir la palette de nos experts. En témoigne aussi le changement de dénomination dont a été l'objet la microflore... devenue microbiote... Cela rend compte bien sûr, avec le suffixe "biote ", de sa diversité et de ses rôles extrêmement divers dans l'immunité,

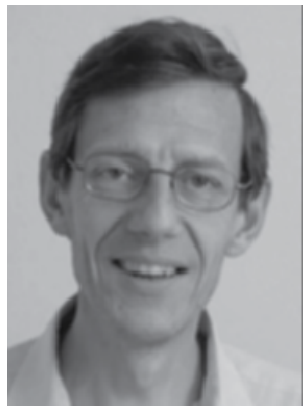
le métabolisme, la santé intestinale.

Il faut dire qu'avec $400 \mathrm{~m}^{2}$ de surface étalée et $10^{14}$ bactéries, ce microbiote comporte plus de cellules et plus de gènes que l'organisme humain : à moins qu'il ne soit qu'un organe délocalisé ou mieux une fonction concédée par un hôte bienveillant !

Les textes proposés vous passionneront, ils vous stupéfieront par leur nouveauté.

Philippe Gérard vous livre les secrets de cette flore, un ami qui vous veut $\mathrm{du}$ bien dès la naissance. Jean-Michel Antoine vous montrera comment elle peut être influencée par les ferments lactiques, ces probiotiques présents naturellement dans tant d'aliments fermentés, le lait en premier lieu. Corinne Grangette et l'équipe de Marie-José Butel vous dévoileront leur rôle dans l'immunité locale, mais aussi dans les défenses immunitaires de tout l'organisme et dans la prévention, voire la prise en charge très précoce de l'allergie. Philippe Marteau vous fera part de l'ensemble des études qui font des probiotiques de véritables alliés dans certaines maladies intestinales chroniques inflammatoires. Marcel Roberfroid, grand précurseur de la notion de prébiotiques, nous dira comment certaines fibres, substrats spécifiques de la fermentation colique, jouent des rôles majeurs pour la santé humaine. Je vous montrerai à quel point la composition de la flore intestinale semble impliquée dans la constitution de lobésité et dans ses complications métaboliques et inflammatoires, et comment sa modulation pourrait avoir des conséquences en termes de prévention, voire d'amélioration du poids. Enfin, Paul Goetz reviendra aux sources en partant non pas des racines, mais d'un tubercule bien délaissé le topinambour, véritable «bombe " prébiotique.

Un dossier qui n'est pas difficile à digérer et qui, une fois de plus, révèle l'extraordinaire complexité de cette discipline qu'est la nutrition, pour le plus grand bien de notre alimentation et de notre santé. 